\title{
Die Österreichische Schule als Gegenprogramm zur Standardökonomik: Kommentar zum Beitrag von Karl-Friedrich Israel und Jörg Guido Hülsmann
}

\author{
Ansgar Belke
}

Online publiziert: 27. Februar 2019

(C) List-Gesellschaft e.V. 2019

(...) the task of economics is to foretell the remoter effects, and so to allow us to avoid such acts as attempt to remedy a present ill by sowing the seeds of a much greater ill for the future. Ludwig von Mises (1981) S. 23

\section{Einführung}

Der weit ausholenden, facettenreichen und sehr konkreten Analyse von Israel und Hülsmann kann man im Rahmen eines Korreferates kaum annähernd gerecht werden. Ich konzentriere mich deshalb wie die Autoren punktuell auf wenige Anwendungsbeispiele aus der Geldtheorie und -politik. Denn der Fokus auf eine monetäre Ökonomie und deren Implikationen zieht sich systematisch durch die Analyse der Vertreter der Österreichischen Schule (Hagemann et al. 2010, S. 183). Zum anderen gehe ich selektiv auf Beispiele aus der Konjunkturtheorie der „Austrians“ ein. Das Bindeglied meiner Kurzanalysen wird die Rolle der empirischen Analyse, und hier insbesondere der Statistik und Ökonometrie, aus der Sicht dieser Denkschule sein.

Um es vorwegzunehmen, die „Standardökonomik“ (der so genannte „Mainstream") und die „Österreichische Schule“ (ÖS) sind meiner Wahrnehmung nach inhaltlich und methodisch viel näher beieinander als es die Aufteilung in Vertreter

This commentary article refers to the article available online at https://doi.org/10.1007/s41025-01900125-8. An author's reply to this commentary article is available online at https://doi.org/10.1007/ s41025-019-00139-2.

\footnotetext{
A. Belke $(\bowtie)$

Jean Monnet-Lehrstuhl für Makroökonomik, Universität Duisburg-Essen, Essen, Deutschland E-Mail: ansgar.belke@uni-due.de

CEPS, Brüssel, Belgien

IZA, Bonn, Deutschland

King's Business School, London, Großbritannien
} 
von Programm und Gegenprogramm in diesem Band des List Forums und der Begriff „Gegenprogramm“ im Titel des Aufsatzes von Israel und Hülsmann insinuieren. Dies gilt erst recht nach meiner Lektüre des sehr anregenden Beitrags von Israel und Hülsmann.

Zumindest in den Bereichen der monetären Ökonomie und der Theorie der Konjunkturzyklen verschwimmen die Unterschiede der ÖS zu dem Teil des so genannten „Mainstreams“, der systematischen Staatsinterventionen keinen grundsätzlichen Freifahrtschein erteilen, bis zur Unkenntlichkeit. Es handelt sich bei diesen Denkschulen demnach nicht um „Programm und Gegenprogramm“. Und auch der Sinn der Einheitsbezeichnung „Mainstream“ für eine ausdifferenzierte Gruppe an Ökonomen sollte deutlich hinterfragt werden. Denn Untersuchungsobjekte wie die Ungleichverteilung von Einkommen zählen zu beider Forschungsprogramm und die Methoden der ÖS zur Teilmenge der Methoden des sogennannten „Mainstreams“. Mit anderen Worten: von dem, was eine Gruppe an „Heterodoxen“ einfordert, gibt es Vieles schon längst. Die Differenzierung in „Standardökonomik“ und ÖS erscheint deshalb nicht zielführend und teils sogar irreführend. Sind es am Ende sogar strategische Gründe, die hierzu führen (Backhouse 2004)?

Wenn man schon unbedingt in Schulen einteilen will, sollte man die Begriffsgegensätze „Österreichische Schule plus nicht per se interventionsoptimistische ,Mainstreamer““ versus ,grundsätzlich interventionsoptimistische ,Mainstreamer““ nutzen. $\mathrm{Zu}$ letzteren sind unbedingt die politisch einflussreichen Anhänger der am MIT Boston lange vertretenen neu-keynesianischen Lehre sowie die Vertreter der „Dynamic Stochastic General Equilibrium (DSGE)“-Modelle zu zählen, aber auch, da ebenfalls politisch einflussreich, anders als Israel und Hülsmann auch viele andere Keynesianer. Da zudem noch nicht einmal klar wird, worauf sich der Begriff „Mainstream“ an sich gründet und warum eine kleine Gruppe hier ein Begriffsmonopol haben sollte, verwende ich in meinen weiteren Ausführungen den Begriff „Mainstream“ nur in Anführungszeichen.

Ich trage mit meinem Korreferat dennoch gerne ein wenig dazu bei, die Gedanken der ÖS stärker als bisher in die theoretische und wirtschaftspolitische Debatte zu integrieren und dort auch zu etablieren. Denn sie ergänzen in der Art und Weise, wie sie vorgetragen werden, den herkömmlichen interventionsskeptischen Teil des „Mainstreams“ recht gut. Zudem schärfen sie gerade bei der Politikevaluation das Profil der Ökonometrie in Richtung einer ,,bescheidenen“ Vorgehensweise, die sich ihrer Beschränktheit bewusst ist.

Der ÖS wird deshalb keinesfalls gerecht, dass Vertreter des neo-keynesianischen „Mainstreams“ die ökonomische Zunft mahnen, die Methode und folglich auch die inhaltlichen Positionen der ÖS nicht zu übernehmen. Diese durchaus nicht seltenen Kommentare atmen den Geist, dass die ÖS geradezu in Heiligenverehrung (Mengers, Hayeks oder Mises) erstarre und diese anrufe, wenn man als Ökonom sonst nicht mehr weiter wisse. Diese stark abwertende Kritik wird zudem regelmäßig genährt durch den Vorwurf der Verwendung einer Verbalökonomik, die nicht auf ausgefeilten formellen Modellen beruhe, und deshalb notwendigerweise willkürlich bleibe.

Auch ist es nicht annähernd zutreffend, die „Österreicher“, wie es häufiger geschieht, ausschließlich als „Neoliberale“ im Sinne der Chicagoer Schule anzusehen. Bezeichnend ist, dass sie, statt sie korrekterweise als ,klassisch liberal“ zu be- 
zeichnen, mit Adjektiven wie „marktradikal“ belegt werden, um sie verächtlich zu machen (Jilch 2016). Zwar teilen die Chicagoer und die ÖS viele Ideen über freie Marktwirtschaft. Anhänger beider Schulen sprechen sich grundsätzlich gegen Preiskontrollen, Produktregulierungen und die staatliche Bereitstellung von Bildung aus. Jedoch bleiben zahlreiche wichtige Unterschiede bestehen. Beispielsweise befürwortet die Chicagoer Schule Zentralbanken und Kartellbehörden, während die ÖS sich dagegen ausspricht (Bagus 2016). Dies lässt sich gut an einem Vergleich von Friedman's „Constant Money Growth Plan“ (Friedman 1960) mit Hayek’s „Competition of Currency Concept" illustrieren (Hayek 1976b, Belke und Polleit 2010, S. 352). Während die „Österreicher“ den Markt nur zu erklären versuchen, wollen Monetaristen wie Milton Friedman ihn steuern - über die Geldmenge.

Die „Österreicher“ wurden von Anfang an sowohl von interventions-optimistischen Fachkollegen, die anders als bei Israel und Hülsmann nicht mit dem gesamten „Mainstream“ gleichzusetzen sind, als auch von der Politik angefeindet. Dies kam nicht überraschend, denn die ÖS argumentierte schon immer recht kühn, dass es zu den Prioritäten eines Ökonomen gehöre, (a) einer Regierung mitzuteilen, was sie alles nicht tun kann, (b) keine Allwissenheit vorzutäuschen und (c) aufzuzeigen, dass Mathematik, Statistik und Ökonometrie in der Ökonomie vor allem bei der Prognose und Politikevaluation eigentlich keinen Platz haben („The equations of mathematical economics are therefore useless for all practical purposes" (Mises 1977, S. 99)).

Diese Sichtweise wurde jedoch von der Geschichte in Gestalt größerer sozialistischer durch Karl Marx motivierte Experimente trotz Mises' Warnungen in den 1920er Jahren zunächst einmal hinweggespült. Den Zusammenbruch des Weltsozialismus 1989 hatte Mises vorhergesagt (Jilch 2016). Die Überzeugung, mit mathematischen Modellen selbst ganze Gesellschaften steuern zu können, ist heute jedoch immer noch weit verbreitet. Notenbanken steuern ihre Geldpolitik auf der Grundlage von DSGE-Modellen. Politiker werden nach wie vor dafür gewählt, dass sie die Schaffung von Arbeitsplätzen versprechen und Politikfolgenabschätzungen wie zum Beispiel von zusätzlichen staatlichen Investitionen werden wie schon immer durch Multiplikatormodelle erstellt. Gegen ihren Widersacher Keynes hatte es die ÖS augenscheinlich schwerer als gegen Marx (Jilch 2016).

Das Anliegen der (Makro-) Ökonomen sollte es sein, mit ihrer Forschung und politischen Beratung dem ,gesellschaftlichen Wohl“" zu dienen. Geeignete wirtschaftspolitische Rezepte für einzelne Länder sind dabei nach den Erfahrungen, die man während der europäischen Schulden- und Bankenkrise mit den Programmländern der Eurozone gemacht hat und auch ich selber im Rahmen von Studien im Auftrag des Europäischen Rechnungshofs zur Effizienz der Troika in den Programmländern gemacht habe, durchaus unterschiedlich (Alcidi et al. 2016). Sie können je nachdem Elemente enthalten, die (so wie es einige unterscheiden wollen) „orthodoxer“ oder „heterodoxer" Prägung sind. Die partiellen Misserfolge des Internationalen Währungsfonds bei dessen weltweiten Rettungsprogrammen beispielsweise sind unter anderem darauf zurückzuführen, dass allen Ländern ein einziges, mit nur einer Denkschule verbundenes „Rezept“ verabreicht wurde (Belke 2014). 
Was zählt, ist also der Wettbewerb der verschiedenen Denkschulen um das beste Konzept, die beste Theorie und Empirie - auch im konkreten Anwendungsfall. Wir können zum Beispiel durchaus empirisch validieren, ob eine Politik der Verringerung überbordender Staatsverschuldung, von einigen wertend als „Austerität“ bezeichnet, im konkreten Fall mehr oder weniger Wachstum bringen wird. Auch Vertreter „heterodoxer“ Ansätze wie die „Österreicher“" sollten sich diesem Wettbewerb stellen und sich nicht dagegen immunisieren, um auch in der praktischen Politik der Marginalisierung zu entgehen.

\section{Die Lucas-Kritik als Bindeglied zwischen „Österreichern“ und dem „Mainstream“6}

\subsection{Hauptaussagen der Lucas-Kritik}

Im Jahr 1976 publizierte Robert E. Lucas seinen wegweisenden Artikel „Econometric Policy Evaluation: A Critique“, in dem er vor einer breiten und unkritischen Anwendung großer ökonometrischer Modelle für die Politikevaluation warnte (Lucas 1976). Sein Hauptargument ist, das Änderungen des wirtschaftspolitischen Regimes, also systematische Änderungen der Ausrichtung der Politik die Struktur des zugrunde liegenden ökonomischen Systems ändern, was die beobachtbaren Beziehungen zwischen den Variablen ebenfalls ändert. Grundsätzlich und eben nicht nur im Fall der großen keynesianischen Makromodelle der 1960er und frühen 1970er Jahre, machen politisch induzierte Regime-Änderungen bisher existierende ökonomische Beziehungen obsolet. Da sich Verhaltensparameter und somit Strukturgleichungen systematisch ändern, kommt es zu Brüchen in den Parametern der reduzierten Form eines Modells (Belke 2000, Belke und Polleit 2010, S. 675). Selbst die sorgfältigste ökonometrische Analyse scheint demnach nicht in der Lage zu sein, eine systematische wirtschaftspolitische Maßnahme zu rechtfertigen. Oft wird übersehen, dass John Maynard Keynes selbst in gewissem Umfang in seiner General Theory und auch an anderer Stelle die von Lucas (1976) thematisierten Probleme analysierte. Deshalb sollten auch Postkeynesianer mit den Inhalten der Lucas-Kritik vertraut sein und diese inhaltlich stützen (Olesen 2016).

Dieses Problem wurde in der Folgezeit auch von weiteren „Mainstream“-Ö̈konometrikern (Christ 1993, S. 77 f., Granger 1999, S. 54, 2003, S. 71) und Makroökonomen anerkannt. In dieser Hinsicht ist der Lucas-Artikel einer der einflussreichsten makroökonomischen Beiträge überhaupt (LeRoy 1992, S. 235). Vor diesem Hintergrund wirkt die Aussage von Israel und Hülsmann: ,Tatsächlich wird das, was man wissenschaftliche Erklärung nennt, in der SÖ im Wesentlichen auf empirische Prognosen reduziert.“ „Phänomene gelten dann als wissenschaftlich erklärt, wenn sie mit einer gewissen Sicherheit vorhergesagt werden können“ geradezu absurd. Hier möchte man sich tendenziell mit einem „Gegenprogramm“ von etwas absetzen, was es so mehrheitlich gar nicht gibt.

In der Tat ist der Weg, den die Makroökonomik nach der Lucas-Kritik seit 1976 beschritten hat, aus der Sicht der ÖS nicht der glücklichste gewesen. Dies lässt sich aber ebenfalls aus der Sicht interventions-skeptischer „Mainstream“-Ökonomen gut 
nachvollziehen. Denn wie Skott (2014, S. 513) aufzeigt, ,(t)he result has been a long and wasteful detour with enormous costs, both in terms of the loss of knowledge in the profession and, more importantly, mistaken policy ... as such ... the straightjacket of full intertemporal optimization misrepresents real-world decision making. It also reduces the ability of the theory to incorporate important aspects of reality in a tractable manner, and therefore encourages the theorist to ignore them".

Dies bedeutet jedoch nicht, dass die Lucas-Kritik grundsätzlich irrelevant ist. Das Gegenteil ist der Fall, so wie es Israel und Hülsmann richtig diagnostizieren. Die ökonomischen Erfahrungen aus mehr als vierzig Jahren nach Veröffentlichung der Lucas-Kritik, wie zum Beispiel der Stagflation in den 1970ern, zeigen nachdrücklich, dass Lucas mit den meisten Punkten seiner im Jahr 1976 vorgelegten Argumentation genau richtig lag. Dies sollte sowohl eine wichtige Lektion sowohl für den interventionsfreundlichen Teil der „Mainstreamer“ als auch für die ,heterodoxen“ Postkeynesianer und „Austrians“ sein.

Israel und Hülsmann führen in diesem Zusammenhang wie Basse (2006, S. 16) Ludwig von Mises an, der genau hier ansetzt und ,... hartnäckig betont, dass es ... empirische ... Konstanten im Wirtschaftsleben nicht gäbe. Seine Arbeiten zu den epistemologischen und methodologischen Grundlagen der Ökonomik können herangezogen werden, um dem Kern der Lucas-Kritik eine neue Interpretation zu geben, nicht als empirische Hypothese, sondern als eine grundsätzliche methodologische Einschränkung der Sozialwissenschaften ....“.

Israel und Hülsmann führen weiter aus, die Lucas-Kritik sollte „nicht lediglich als eine Kritik der älteren ökonometrischen Makromodelle angesehen werden, sondern vielmehr als ein warnender Hinweis, der a priori Gültigkeit beansprucht. Jedes quantitative ökonomische Modell, das sich auf konstante funktionale Beziehungen zwischen messbaren Größen des Wirtschaftslebens stützt, um die Konsequenzen bestimmter politischer Interventionen zu simulieren, sei anfällig für diese Kritik“. Dies ist jedoch, wie einführend bereits erläutert, eindeutig keine neue Interpretation der Lucas-Kritik, sondern gibt nur die von Lucas (1976) selbst vorgebrachte Verallgemeinerung wieder. Stattdessen tun Israel und Hülsmann so, als ob die ÖS die Lucas-Kritik von einer angeblichen Verengung auf die Kritik an den großen keynesianischen Modellen befreien könnte. Hier wird jedoch offensichtlich ein unnötiger „Strohmann“" aufgebaut.

Dass, wie es Israel und Hülsmann ausführen, ,keine noch so genaue empirische Untersuchung Sicherheit darüber gewähren kann, ob ein bisher relativ zuverlässiges Modell auch in der Zukunft noch brauchbare Prognosen abliefert", liegt in der Natur der Sache und könnte deshalb als ein Allgemeinplatz bezeichnet werden. Gerade deshalb werden ja stochastische Modelle angewendet und gewisse Eintrittswahrscheinlichkeiten angesetzt. Die These der Verfasser, dass ,(s)tattdessen die Empirie bisher für jedes ökonomische Modell aufgezeigt (hat), dass die angenommenen funktionalen Zusammenhänge und die geschätzten Parameter über die Zeit nicht konstant bleiben" ist schlicht nicht korrekt. Denn auch hier liegt die Wahrheit in der Mitte: es gibt „Great ratios“ zwischen makroökonomischen Variablen, die selbst längerfristig konstant sind, und solche, die es eben nicht sind. 


\subsection{Neue Version der Lucas-Kritik: Kann sie „Austrians“ und ,Mainstreamer“6 vereinen?}

Selbst Vertreter der ÖS, die normalerweise argumentieren, dass formale ökonomische Modelle die Komplexität dynamischer Marktprozesse grundsätzlich nicht hinreichend erfassen können, tendieren dazu, die Lucas-Kritik, wenn auch in neuer Form ohne die Annahme rationaler Erwartungen, zu akzeptieren (Basse 2006 und der hier zu diskutierende Beitrag von Israel und Hülsmann). Es wäre also logisch konsistent und anreizkompatibel, wenn die „Österreicher" eine neue und kraftvollere Version der Lucas-Kritik vertreten würden, die einerseits nicht länger auf der Theorie rationaler Erwartungen beruht, aber andererseits dann auch nicht mehr allein durch die These in Frage gestellt werden kann, dass der Ansatz rationaler Erwartungen fragwürdig sei.

An dieser Stelle der Analyse sollte man es aber nicht versäumen, einschränkend Mises erneut zu zitieren: „In the field of human action, however, there are no such constants. The equations of mathematical economics are therefore useless for all practical purposes“ (Mises 1977, S. 99). Da es in der Ökonomie keine zeitlich konstanten Beziehungen gebe, könne die Ökonometrie nur auf dem Gebiet der Wirtschaftsgeschichte sinnvoll angewandt werden (Basse 2006). Die folgenden Abschnitte widmen sich der Frage, wie vielversprechend trotzdem die Entwicklung einer neuen ,österreichischen“ Variante der Lucas-Kritik ist.

\section{3 „Austrian School“"-kompatible Ökonometrie}

Die Forschungsprogramme der Vertreter der ÖS und der „Mainstream“-Ökonomen, die nicht der (Neo)keynesianischen Denktradition angehören und deshalb auch auf die systematische Simulation von Politikfolgen verzichten, ähneln sich im Hinblick auf die Befolgung der Prinzipien der Lucas-Kritik sehr stark. Die Vertreter der ÖS könnten zwar theoretisch die Mises-Version der Lucas-Kritik wie sie in Abschn. 2.2 kurz dargestellt wurde, als zusätzliches Argument anführen, ihre Aversion gegenüber formaler Modellierung und der Ökonometrie zu begründen. Aber ohne empirische Evidenz wird eine „österreichische“ Version der Lucas-Kritik nicht viele „Mainstream"-Ökonomen von der Begrenztheit der traditionellen Lucas-Kritik überzeugen. Daher sollte die ÖS aus strategischer Sicht eine etwas andere Forschungsstrategie als bisher verfolgen.

Ökonomen der ÖS könnten somit im Einklang mit Prinzipien der ÖS durchaus historische ökonomische Ereignisse empirisch analysieren und die Relevanz der Lucas-Kritik empirisch testen. Sie sollten sich der Ökonometrie nicht mit Verweis darauf, dass jegliche Ökonometrie im Mises'schen Sinne notwendigerweise den Einstieg in die Planwirtschaft bedeute, vollständig enthalten. Nach Mises können sich ökonometrische Analysen aber lediglich auf die Vergangenheit (,economic history“), aber nicht auf die Zukunft (,forecasts“ oder „simulations“) beziehen. Und das tun sie offensichtlich auch.

Ökonomen der ÖS präsentieren immer wieder historische Evidenz, um ihre Befunde zu illustrieren. Nicht wenige von ihnen nutzen diese sogar im Widerspruch zu Mises in der Praxis, um für substanzielle Änderungen des Kurses der Wirtschaftspoli- 
tik wie zum Beispiel einen sofortigen Ausstieg aus der unkonventionellen Geldpolitik zu argumentieren (Abschn. 4). Die meisten Vertreter der ÖS akzeptieren folglich, dass statistisch-ökonometrische Verfahren zumindest verwendet werden dürfen, um die Vergangenheit zu verstehen. Dies belegt auch das folgende Zitat von (Hoppe 1997, S. 74): ,[A]lthough questions of this nature may easily degenerate into idle semantic quibbles such as whether a glass of water is half empty or half full, empirical questions - disagreements on matters of fact - are accessible to empirical research and can, in principle, be decided upon based on the observation of the facts".

Mit ihrer Denkschule kompatibel wäre somit die Durchführung von Strukturbruchtests (Chow-Tests, rekursive Koeffiziententests, CUSUM-Tests) im Rahmen von Analysen schwacher und starker Exogenität, um zu zeigen, dass strategische Änderungen der Wirtschaftspolitik Strukturbrüche in den relevanten Zeitreihen, aber auch - wie im Hysterese-Fall - in der Beziehung zwischen diesen Variablen geführt haben (Belke 2000; Belke et al. 2014). Auf diese Weise könnte ökonometrische Forschung in stärker „Österreichischer“ Weise durchgeführt werden, um interventionsfreundliche „Mainstream“-Ökonomen von den Gefahren einer unkritischen Anwendung ökonometrischer Methoden zu überzeugen.

Einige Vertreter dieser Denkschule mögen dieser Forschungsstrategie skeptisch gegenüberstehen. Denn nach Mises ,there are no constants in the field of human action“ (Mises 1962). Ökonomische Akteure verhalten sich jedoch in vielerlei Hinsicht recht stabil (,consumption smoothing“ etc.). Folglich könnte es gerechtfertigt erscheinen anzunehmen, dass die Koeffizienten eines Regressionsmodells, das das Verhalten ökonomischer Akteure ohne Strukturbrüche beschreibt, ebenfalls über die Zeit hinweg mehr oder weniger stabil sind. Die Gültigkeit dieser Annahme kann mit Tests der Parameterkonstanz getestet werden. Ein Regressionsmodell ist natürlich nicht verwendbar, falls diese Tests Anzeichen von Parameterinstabilität anzeigen. Andernfalls kann es jedoch als ein Instrument zur Analyse historischer Ereignisse verwendet werden.

Dieses Vorgehens ist für den „Mainstream“ in gleicherweise wie aus Sicht der ÖS legitim. Deshalb lässt sich nicht nachvollziehen, warum einige aktuelle Ökonomen der ÖS die Anwendung statistisch-ökonometrischer Testverfahren für die Analyse historischer Evidenz generell nicht akzeptieren und warum sie es ablehnen, empirisch zu prüfen, ob ihre theoretischen Betrachtungen vergangene Ereignisse erklären können (Backhouse 2004; Basse 2006, S. 22).

\subsection{Lucas-Kritik - Strategische Aspekte aus der Sicht der Österreichischen Schule}

Es wäre in der Tat hilfreich, wenn ökonometrische Tests der neuen Version der Lucas-Kritik auch die interventionsfreundlichen „Mainstream“-Ökonomen letztlich davon überzeugen könnten, dass staatliche Intervention nicht durch eine Untersuchung der Vergangenheit gerechtfertigt werden kann. Obgleich die traditionelle Lucas-Kritik sehr einflussreich war, wurde ihre allgemeine Akzeptanz durch die für sie konstitutive Theorie rationaler Erwartungen eingeschränkt. Wäre die Gültigkeit der Lucas-Kritik jedoch nicht an die enge und umstrittene Annahme rationaler Erwar- 
tungen gebunden (,neue Version“ der Lucas-Kritik), dürften es interventionsfreundlichen „Mainstream“-Ökonomen ungleich schwerer haben, die „Österreichische“ Version der Lucas-Kritik zu verwerfen oder ganz zu diskreditieren (Basse 2006, S. 22). Darauf zu verzichten, auf der Grundlage einer kraftvollen, modifizierten Version der Lucas-Kritik empirische Evidenz zu liefern, um interventionsfreudige „Mainstream“-Ökonomen vom Gegenteil zu überzeugen, wäre im Hinblick auf die wirtschaftspolitischen Hauptanliegen der ÖS sicherlich riskant und taktisch unklug. All dies gehört jedoch schon länger zum Arsenal vieler interventionsskeptischer „Mainstream“-Ökonomen und ist eigentlich nichts ÖS-spezifisches.

Beide Versionen der Lucas-Kritik untersagen die Verwendung der Ökonometrie zur Rechtfertigung wirtschaftspolitischer staatlicher Interventionen, da diese notwendigerweise zu Strukturbrüchen führen. Ironischerweise verwenden gerade „Mainstream“-Ökonomen, zumindest wenn sie zu der interventionsfreundlichen Teilgruppe gehören, ökonometrische Methoden und folgen dabei eben nicht den Leitlinien der Lucas-Kritik. Ökonomen der ÖS hingegen sind grundsätzlich gegen jegliche Staatsintervention und wenden, wenn sie prinzipienfest sind, keine ökonometrischen Analysen an, um wirtschaftspolitische Maßnahmen zu rechtfertigen. Viele dürften sogar grundsätzlich von der Verwendung ökonometrischer Methoden Abstand nehmen. Aus dieser Sicht ist ein Ökonom der ÖS im Prinzip auch ein idealer Ökonometriker. „Austrians“ betonen durchweg, dass die ökonometrische Analyse nur bei der Analyse der Vergangenheit zielführend ist, jedoch nicht zur Prognose zukünftiger Ereignisse verwendet werden kann (Basse 2006, S. 23).

Gerade diese kritische Attitüde der ÖS gegenüber der Ökonometrie ist einer trennscharfen und klugen ökonometrischen Forschung sehr förderlich: „Austrian economists, for example, surely never would dare to run a regression without prior thinking about economic theory“ (Basse 2006, S. 23). Eine von „Österreichern“ durchgeführte profunde ökonometrische Analyse würde sicherlich dazu beitragen offenzulegen, dass eine Vielzahl von Vertretern aktivistischer staatlicher Interventionen ihre teils fragwürdigen Ideen regelmäßig hinter ganzen Batterien kryptischer Teststatistiken verstecken.

\subsection{Kointegrationsanalyse und empirische Hysterese-Forschung ,österreichischer" Prägung}

In der Tat kann die Ökonometrie ein sehr wirkmächtiges Forschungs-Tool sein, solange bestimmte grundsätzliche Regeln beachtet werden. „Austrians“ sollten deshalb definitiv nicht akzeptieren, dass dieses wirkmächtige Instrumentarium ausschließlich von interventionsfreundlichen ,Mainstream“-Ökonomen genutzt wird, die tendenziell gegen die Lucas-Regeln verstoßen und Politikmaßnahmen mit ihren empirischen Arbeiten rechtfertigen.

Zahlreiche Ökonometriker haben bereits seit geraumer Zeit damit begonnen, die Art und Weise in Frage zu stellen, mit der Ökonometrie in der Vergangenheit durchgeführt wurde. Bezeichnend dafür ist unter anderem die Nobelpreisvergabe an Robert F. Engle und Clive W.J. Granger im Jahr 2003. Es scheint sogar gerechtfertigt zu schlussfolgern, dass ,,(t)oday many econometricans seem to be ready to understand the Austrian message“ (Basse 2006, S. 23). Es etablierte sich in diesem Zuge 
eine neue, fruchtbare und einflussreiche Technik in der Ökonometrie, die Kointegrationsanalyse. Diese Technik basiert auf der Idee, dass in der langen Frist mehr oder weniger stabile Langfristbeziehungen zwischen mehreren Variablen existieren. Strukturbrüche in dieser Langfristbeziehung lassen sich ohne weiteres berücksichtigen. In der kurzen Frist können sich die empirischen Ausprägungen der Variablen hingegen von der Gleichgewichtsbeziehung wegbewegen. Falls die Variablen jedoch miteinander kointegriert sind, existiert ein Mechanismus oder ein Prozess, der sie wieder zurück in die Gleichgewichtsbeziehung bringt. Hier gibt es starke inhaltliche Parallelen zu makroökonomischen Gleichgewichtskonzepten wie die Natural RateHypothese der Arbeitslosigkeit (Belke 2000).

Die Kointegrationsanalyse liefert eine neue und wahrscheinlich deutlich „österreichisch " geprägtere Interpretation von Gleichgewichtssituationen (Granger 1991, S. 68). Die Technik der Kointegrationsanalyse stellt ein interessantes Werkzeug dar, um historische Daten zu untersuchen, wenn man wie die ÖS daran interessiert ist, Marktprozesse zu studieren. Diese Ideen tragen einen genuin „österreichischen“ Charakter. Denn die Vertreter der ÖS argumentierten immer schon, dass ökonomische Handlungen in der Zeitdimension getätigt werden und dass die neoklassische Idee des Gleichgewichts ohne Bezug auf die Zeitachse nicht zielführend sei: „Regaining a healthy respect for the temporal element requires that we look at the market process that transforms a sequence of short runs into a long run" (Garrison 1986, S. 90).

Einschränkend ist anzumerken, dass die vorstehenden Argumente nicht in Bezug auf nicht-strukturelle Vektorautoregressive (VAR) Modelle (die Gleichungen in reduzierter Form enthalten, McCallum 1982, S. 10, sowie Sargent 1979, S. 8 und 14) und streng genommen auch nicht für „Granger-Kausalitätsanalysen“ (Garrison 2018, Granger und Newbold 1977, S. 225) gelten. Beide sind anfällig für die ursprüngliche und auch die modifizierte Lucas-Kritik.

Die Kointegrationsanalyse interpretiert kurzfristig auftretende Ungleichgewichte als Phänomene, die zu Anpassungsprozessen führen. Somit kommt sie einer empirischen Untersuchung von temporären Elementen des Marktprozesses gleich. Sie kann somit als eine Modellierungsstrategie aufgefasst werden, die Kurzfristdynamik und langfristige Gleichgewichte miteinander kombiniert. Man kann sie sogar in „österreichischer“ Tradition nutzen, um Zeitpfadabhängigkeiten (Hysterese) im Sinne sich mit jedem Schock ändernder Kointegrationsbeziehungen abzubilden (Belke 2000; Belke et al. 2014). Es geht dann um zeitpfadabhängige Gleichgewichtsbeziehungen, die von der ÖS durchaus thematisiert wurden (siehe zum Beispiel John Charalambakis 2016). Beispielsweise wurde das Konzept der ÖS mit der Zeit als Produktionsfaktor von Schumpeters Schüler Georgescu-Roegen (1971) in das „Flow Fund Model in the physics of hysteresis" (Shmelev und Shmeleva 2012) integriert.

Schließlich wurden in jüngster Zeit im Rahmen der bayesianischen Kointegrationsanalyse Schätzverfahren mit zeitvariierenden Koeffizienten entwickelt (Belke et al. 2011; Koop 2017). Bereits Caplan (2003) hatte „Bayes’ Rule“ als ein Heilmittel gegen „methodological schizofrenia“ empfohlen (vgl. anders aber Block 2005, der im Rahmen einer Mini-Kontroverse die Frage erhob: „Why do economists react so violently to empirical evidence against the conventional view of the minimum wage's effect?" und sie mit der Feststellung beantwortete, „that economists do so 
because they are covert praxeologists"). Folglich könnte es die Technik der Kointegrationsanalyse selbst Ökonomen der ÖS ermöglichen, ökonometrische Methoden anzuwenden, ohne ihre zentralen Themen opfern zu müssen (Basse 2006). Dies gilt zumindest so lange, wie man auf Prognosen auf Basis dieser Schätzungen verzichtet.

\subsection{DSGE-Modelle als erfolgreiche Reaktion auf die ursprüngliche Lucas- Kritik?}

In der Makroökonomik werden aktuell die mikrofundierten Allgemeinen Stochastischen Gleichgewichtsmodelle (DSGE) in ihrer Neukeynesianischen oder Neo-Wicksellianischen Variante (Woodford 2003; Galí 2008) bei der Analyse von Geldpolitik als eine Reaktion auf die Lucas-Kritik angeboten. Nach Galí und Gertler (2007, S. 27) sei es mit ihnen anders als mit älteren Makromodellen möglich, verlässliche kontrafaktische Politikevaluierungen durchzuführen. Israel und Hülsmann haben jedoch vollkommen Recht, dass der Nutzen der DSGE-Modelle stark von einer plausiblen Identifikation struktureller Parameter und der Konstanz empirischer Beziehungen abhängt (siehe auch Israel 2016).

DSGE-Modelle liefern zwar einen kohärenten Analyserahmen. Diese Kohärenz wird aber durch eine unzulässige Beschränkung des Verhaltens ökonomischer Akteure auf eine dynamische Nutzenmaximierung und rationale Erwartungen erkauft (De Grauwe 2010; Israel 2016). Das Problem der DSGE-Modelle besteht in der Tat darin (und auch hier stimme ich mit Israel und Hülsmann überein), dass sie außerordentliche kognitive Fähigkeiten individueller Agenten unterstellen. Darüber hinaus benötigt diese Modellgattung eine ganze Reihe an ad hoc-Annahmen, um die Modelle mit den Daten in Einklang (,Fit“) zu bringen (De Grauwe 2010). Diese führen aber letztlich dazu, dass das, ,was man hineinsteckt, auch wieder herauskommt“ (,the inclusion of priors also helps identifying parameters“, Romer 2016, S. 14, Olesen 2016). Schließlich fragen sich viele „Mainstream“-Ökonomen, wie man mit DSGE-Modellen geldpolitische Analysen durchführen soll, wenn in ihren Varianten gar kein „Geld“, sondern nur der Zins enthalten ist. Wieviel Sinn macht es, wenn die Europäische Zentralbank auf der Grundlage eines bayesianischen DSGE-Modells nach Smets und Wouters (2003) mittelfristige Inflations-Projektionen erstellt. Denn das Modell tendiert ohnehin zu einem eindeutigen Gleichgewicht.

Ein möglicher Ausweg bestünde darin, Modelle zu entwickeln, die beschränkte kognitive Fähigkeiten der Akteure anerkennen. Rationalität ließe sich dann immer noch durch ein „trial and error“-Lernen berücksichtigen. De Grauwe (2010) beispielsweise entwickelt ein derartiges Modell und analysiert dessen Implikationen (vgl. auch Olesen 2010, 2016).

Insgesamt gesehen werden die DSGE-Modelle gleichermaßen von Vertretern der ÖS und des „Mainstreams“, nicht nur vertreten durch Paul Romer (2016), harsch kritisiert. Romer (2016, S. 8 und 11) bezeichnet eine DSGE-Modellbasierte Makrookonomie wegen der nicht gelösten Identifikationsproblematik und der Nichtberücksichtigung von „Real life“-Phänomenen vernichtend als ,,post-real“ macroeconomics“. Auch hier liefert die ÖS wieder kein klares Gegenmodell zum „Mainstream“. Man findet sich auch hier tatsächlich wieder ,in einem Boot“" wieder. 
Es ist nicht ausgeschlossen, dass DSGE-Modelle vor diesem Hintergrund nur eine „Strohpuppe“ als „Whipping boy“ der Heterodoxen darstellen. Denn nur eine relativ kleine, hoch spezialisierte makroökonomisch orientierte Community arbeitet tatsächlich mit derartig mathematisierten Modellen - dies aber neben dem akademischen Sektor hauptsächlich im Research internationaler Organisationen. Trotzdem dienen sie Vertretern der „Heterodoxie“ häufig als Grundlage für Kritik am gesamten „Mainstream“: mit diesen Modellen sei beispielsweise die Finanzkrise 2008 nicht vorhergesagt worden. Denn, wie es Israel und Hülsmann treffend wiedergeben, ,,sind aus Sicht der ÖS mathematische Gleichungen ... kein notwendiges Werkzeug kausalwissenschaftlicher Forschung“. Hieraus folgt direkt, dass Kritik an DSGE-Modellen weder notwendig, noch hinreichend für fundierte Kritik am, von den Verfassern nicht hinreichend differenzierten, „Mainstream“ ist. Ein Alleinvertretungsanspruch, wie er bei Vertretern der ÖS häufig und auch bei Israel und Hülsmann gelegentlich durchscheint, ist fehl am Platz.

\subsection{Wettbewerb der makroökonomischen Modelle als Leitbild}

Wie in der Einleitung schon formuliert gibt es mehr als nur genau einen Weg, makroökonomische Theorie mikroökonomisch zu fundieren - über Akteure mit perfekter Voraussicht, über einen repräsnetativen Agenten oder über die Modellieung von Haushalten und Unternehmen mit ,bounded rationality“. Entsprechend existiert auch nicht nur ein eindeutiges, einziges makroökonomisches Modell ,for all seasons“. Und es gibt mehr als genau einen eindeutigen Weg, relevantes Wissen über makroökonomische Phänomene und makroökonomische Anpassungsprozesse zu erlangen. Oder wie es Skott (2014, S. 503) ausdrückt: „There can be no single, correct theory or model of ,the economy“. The economy is not a well-defined object and, even if it were, a theory does not aim to provide a complete picture of reality ... Equally self-evident ... is the claim that there can be no single, correct method for gaining insights into the operation of the economy“. Wir benötigen folglich einen Wettbewerb der Methoden.

\section{Gegenentwürfe zur Standardökonomik der Österreichischen Schule? Ausgewählte Beispiele}

\subsection{Taylor-Reaktionsfunktionen und optimale Geldpolitik}

Die von Israel und Hülsmann benannten Probleme, dass zum Beispiel die natürliche Zinsrate und die Produktionslücke nicht-beobachtbare Konzepte sind, sind keinesfalls neu und mittlerweile allseits bekannt. Wie die Autoren selber zugeben, wirft dieser Denkansatz zahlreiche Probleme auf, die sich nicht nur vom Standpunkt der $\ddot{O} S$ aus stellen (für ein ausführliches Beispiel mit ähnlichen Argumenten siehe Belke and Polleit (2010, S. 723-729)). Hier geht die Analyse der ÖS nicht über die schon länger vorhandenen Argumentationsmuster derjenigen Autoren des „Mainstreams“ hinaus, die skeptisch gegenüber dem systematischen Einsatz der Taylor-Regel zur 
Prognose zukünftiger Leitzinsen sind. Es werden stattdessen von den Verfassern leider wieder alle „Mainstreamer“ „,in einen Topf geworfen“.

In Bezug auf die Ableitung einer optimalen Geldpolitik spezifizierte Hayek (1935, p. 27) eine optimale Geldpolitik als eine Politik, die die effektive im Umlauf befindliche Geldmenge unverändert lässt und so die Änderungen des durchschnittlichen Preisniveaus, die aus Änderungen der Geldnachfrage resultieren, stabilisiert (Belke und Polleit 2010, S. 678f.). Hayek kombiniert hierdurch letztlich Elemente der ÖS mit „Mainstream“-Varianten der Quantitätstheorie (Selgin 1990, 1997). Auch in diesem Fall bildet die ÖS also kein reines „Gegenstück“ zum „Mainstream“.

\subsection{Empirische Analyse des Kontrafaktums}

Israel und Hülsmann argumentieren weiter: „Nur der Verlauf der Geschichte, wie er sich tatsächlich manifestiert, kann empirisch, statistisch und quantitativ untersucht werden. Die kontrafaktischen Alternativen bleiben einer solchen Analyse verschlos-

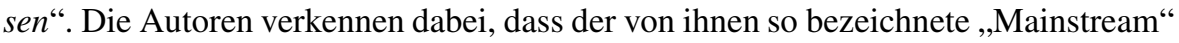
durchaus robuste Analyseverfahren der kontrafaktischen Alternativen entwickelt hat, indem nach strengen statistisch-ökonometrischen Kriterien „,country clones“, d.h. gewichtete Durchschnitte von Ländern, die kein „Treatment“ erfahren haben, als Kontrollgruppe abgeleitet werden (Abadie et al. 2014; Campos et al. 2014). Dies bedeutet, dass kontrafaktische Alternativen prinzipiell konstruierbar sind und durchaus einer historischen empirischen Analyse unterzogen werden können.

\subsection{Definition des Zinses - realwirtschaftliches oder monetäres Phänomen?}

Die Schlussfolgerung des (neo-)keynesianisch orientierten „Mainstreams“ ist, dass der Zinssatz - unter der Voraussetzung, dass die Regierung das Monopol über das Geldangebot hält - als eine Politikvariable anzusehen ist. Durch die Beeinflussung des Zinses können demnach Investitionen, der Output und und die Beschäftigung durch die Regierung beeinflusst werden. Der Einfluss der keynesianischen Zinstheorie auf die Makroökonomik und auch auf die makroökonomische Politik reichte bislang tief. Immer noch ziehen es die Notenbanken vor, kurzfristige Zinsen zu steuern anstatt die Menge an Basisgeld und hierauf aufbauend die Geldmenge zu beschränken, um auch zukünftig nach Normalisierung der Geldpolitik den Tauschwert des Geldes bewahren zu können und Wirtschaftswachstum und Beschäftigung zu fördern (Belke und Polleit 2010, S. 152).

Eine derartige Sichtweise steht in starkem Kontrast zu den „Austrians“ und den zahlreichen „Mainstream“-Ökonomen, die argumentieren, dass die zukünftige erwarteten Steuerbelastungen und Strukturreformen sowie das zukünftig erwartete Wachstum wichtigere Determinanten für die Investitionsnachfrage als der Zins sind - sowohl in der Theorie als auch in Bezug auf praktische und wirtschaftspolitische Implikationen (Belke und Polleit 2010, S. 152). Zum Beispiel argumentierte Ludwig von Mises, dass der Zins ,,is neither the impetus to saving nor the reward or the compensation granted for abstaining from immediate consumption. It is the ratio in the mutual valuation of present goods against future goods." (Mises 1996, S. 527). Und Vertreter des interventionsskeptischen „Mainstreams“ argumentierten 
in genau derselben Weise gegen die Einführung eines Negativzinses im Rahmen der unkonventionellen Geldpolitik und gegen das von interventionsfreundlichen ,Mainstreamern“ vorgebrachte Argument, dass die aktuelle Phase extrem geringer Zinsen das beste Argument für eine staatliche Investitionsoffensive sei. Auch hier lassen sich also wieder große Gemeinsamkeiten zwischen der ÖS und signifikanten Teilen des Mainstreams identifizieren - der Zins wird von beiden Denkschulen als ein realwirtschaftliches und eben nicht primär als monetäres Phänomen gesehen (Belke und Polleit 2010, S. $151 \mathrm{f}$.).

\subsection{Kosten von Inflation}

Ähnliches gilt auch für die Ableitung der Kosten von Inflation - auch hier sind die Schnittmengen der ÖS und dem „Mainstream“ groß (siehe ausführlich Belke und Polleit 2010, S. 381). Die Verbraucherpreisinflation fiel bereits in den 1980er und 1990er Jahren in den meisten westlichen Industrieländern deutlich (Dowd 1994). Dieser Trend setzte sich nicht zuletzt wegen der Übernahme der Inflations Targeting-Strategie durch nahezu alle führenden Notenbanken bis zur Finanzkrise fort. Anschließend wurden sogar Deflationsszenarien diskutiert. Dies erklärt, warum ökonomische Analysen der Inflation immer weniger das Problem hoher Inflation adressieren und stattdessen ihren Fokus auf die Kosten und Nutzen der weiteren Verringerung der Inflation in Richtung Null richten (Belke und Polleit 2010, S. 381).

Die wissenschaftliche Literatur hierzu versucht die Natur dieser Kosten und Nutzen zu spezifizieren und eine Indikation der hiermit verbundenen empirischen Größenordnungen zu vermitteln (als ein Beispiel vgl. Akerlof et al. 1996). Eine erhebliche Einschränkung der Aussagekraft dieser Studien besteht darin, dass sie sich bei der Ableitung der Kosten der Inflation fast ausschließlich auf die Art und Weise, wie Inflation die Geldhaltung beeinflusst, fokussieren (Bailey 1956). Denn diese Perspektive erweist sich bei näherem Hinschauen als viel zu eng (Belke und Polleit 2010, S. 381).

Deshalb machen neuere Entwicklungen in der Theorie-Landschaft der letzten Jahrzehnte eine Neueinschätzung der Kosten von Inflation zu einem verdienstvollen Unterfangen (Horwitz 2003). Insbesondere die Etablierung des Public Choice-Ansatzes, das Aufkommen des „New Institutionalism“-Ansatzes und das Wiederaufleben der „Austrian School of Economics“ haben bei der Abschätzung der Kosten von Inflation zu einer gründlicheren Berücksichtigung der Funktionsweise von Märkten und Institutionen und einer Interaktion zwischen ihnen geführt (Belke und Polleit 2010, S. 381). Alle drei Ansätze gehen also Hand in Hand.

Die ÖS ist also auch aus dieser Perspektive kein „Gegenstück“ zu anderen Denkrichtungen wie dem interventionsskeptischen „Mainstream“. Beispielsweise gibt es substanzielle und lange etablierte Gemeinsamkeiten zwischen „österreichischen“ Ideen und der Public Choice-Literatur. Jedoch stellt die Mehrzahl der Beiträge eher eine Kritik an den Ideen der Public Choice-Theorien als die Entwicklung einer unabhängigen „österreichischen“ Public Choice-Theorie dar (Holcombe 2015). Vertreter der Public Choice-Theorie analysieren ,,collective decision-making“-Prozesse. Ökonomen der ÖS hingegen definieren Aspekte des ,,collective decision-making“ einfach hinweg, um sich auf Probleme des beschränkten Wissens, die eine effiziente Alloka- 
tion von Ressourcen durch Regierungen verhindern, zu fokussieren. Aus diesem und auch anderen Gründen bleibt die Analyse des kollektiven Entscheidungsprozesses durch Vertreter der ÖS (noch) unterentwickelt. Die ÖS beinhaltet jedoch durchaus viele Einsichten, die für die zukünftige Entwicklung einer ,österreichischen“ Public Choice-Theorie verwendet werden könnten (Holcombe 2015).

\subsection{Unsicherheit als zentrales Thema der „Österreicher“" und des ,Mainstreams“6}

Die Aussage von Israel und Hülsmann: „Das grundsätzliche Problem aber, dass es beim Phänomen der Konjunkturzyklen zu erklären gilt, ist nicht Unsicherheit über die Zukunft in Anbetracht dieser Faktoren ..." erscheint zu apodiktisch. Natürlich zeigen die Erfahrungen mit den jüngsten Krisen, dass selbst temporäre Erhöhungen politischer, finanzmarktbezogener und anderer Unsicherheit ganze Volkswirtschaften auf neue „schlechtere“ Trajektorien dauerhaft geringeren Wachstums führen können („Hysterese bei Unsicherheit“). Streiten lässt sich aber sehr wohl darüber, wie man die hierfür relevante Unsicherheit abgrenzt. Ist es der Teil der Unsicherheit, der durch GARCH-Schätzungen u. a. quantifiziert aber dann auch abgesichert werden kann - also das Risiko? Oder ist es im Sinne Frank H. Knights (1964, [1921]) genau der Teil, der nicht gemessen werden kann? „Austrians“ dürften eher der zweiten Sicht zuneigen; innerhalb des Mainstreams gibt es wohl beide Strömungen (Belke und Polleit 2010, S. 480 f.). Legendär ist in diesem Zusammenhang wohl der Friedman'sche Hubschrauber, dessen pures Erscheinen trotz Geldabwurfs der Bevölkerung Angst einjagt und wegen steigender Unsicherheit zu Kaufzurückhaltung führt (Belke 2018). Insgesamt gesehen ist der Grad der Gemeinsamkeiten zwischen ÖS und „Mainstream nicht ganz so groß wie in den zuvor behandelten Fallbeispielen.“

Knight spricht von nichts Geringerem als dem Versagen des Konzepts der Wahrscheinlichkeitsrechnung. In seinem wegweisenden Werk „General Theory of Employment, Interest and Money“ nimmt John Maynard Keynes eine ähnliche Position wie die „Österreicher“ ein (Keynes 1936, S. 161): „,[Most of our decisions] to do something positive ... can only be taken as a result of animal spirit ... and not as the outcome of a weighted average of quantitative benefits multiplied by quantitative probabilities." In der Tat betonte Knight, dass die Schwierigkeiten des Vorhersageprozesses weit über die Unmöglichkeit der Anwendung mathematischer Sätze für Vorhersagen hinaus reicht. Er bestand darauf, dass ,,a priori reasoning“ die Unbestimmtheit der Zukunft nicht eliminieren kann (Belke und Polleit 2010, S. 81).

\section{Die Österreichische Schule in der wirtschaftspolitischen Praxis}

Der Ausgangspunkt und die selbst auferlegte Einschränkung wirtschaftspolitischer Überlegungen der ÖS besteht im Allgemeinen darin, dass sich das menschliche Handeln nicht wie die Erkenntnisobjekte in der Naturwissenschaft erklären oder anhand von „externen Faktoren“ prognostizieren lässt (Polleit 2017). In ihrem Abschnitt „Analytische Schwerpunkte im Vergleich“ postulieren Israel und Hülsmann für die 
Wirkungen einer expansiven Geldpolitik allerdings umfassende und erstaunlich konkrete Verlaufsmuster. Diese könnten inhaltlich gesehen auch ein zentraler Bestandteil vieler gängiger „standardökonomischer“ Analysen sein. Dies zeigen Belke und Polleit (2010) auf breiter inhaltlicher Basis.

Die Ableitung umfassender Wirkungsketten ist typisch für die ÖS, wenn nicht über historische empirische Belege, dann auf praxeologischer Grundlage. Überzeugende Ableitungen ,nicht widerlegbarer Wahrheiten“ im Sinne Immanuel Kants in den Bereichen der monetären Ökonomik und der Konjunkturtheorie sind zwar noch nicht dokumentiert. Trotzdem kommt es immer wieder zu einer starken Einmischung von „(New) Austrians“ in aktuelle makroökonomische Politikdebatten.

Dabei fehlt es aber hin und wieder an Überzeugungskraft, weil lange und komplexe Wirkungsketten ,durchdekliniert“ und für das konkrete wirtschaftspolitische Problem als relevant erklärt werden, ohne konsequent auf die empirische Relevanz dieser einzelnen Teilschritte für die konkrete Analyseperiode einzugehen. $\mathrm{Zu}$ allem Überfluss werden im Widerspruch zu den in Abschn. 2 ausführlich erläuterten Selbstbeschränkungen der ÖS von ihren Vertretern hieraus noch konkrete Politikempfehlungen abgeleitet.

Diese Problematik soll im Folgenden anhand der beiden Themen „Unkonventionelle Geldpolitik und unvermeidliche Inflation“ und „Ultra-lockere Geldpolitik und gemeinschaftliche Haftung“, in die sich Vertreter der ÖS in letzter Zeit konkret zu Wort meldeten, genauer eingeordnet werden.

\subsection{Unkonventionelle Geldpolitik und unvermeidliche Inflation}

Ludwig von Mises leitete bekanntlich drei Phasen der „,monetary destruction “ ab (Rothbard 2010, S. 68-72). In Phase Eins inflationieren die Notenbanken die Währung. Die Öffentlichkeit erwartet jedoch noch nicht, dass die Preise steigen oder sogar, dass sie fallen. Sie halten sich deshalb mit ihren Ausgaben zurück und erhöhen ihre Geldnachfrage.

Phase Eins kann eine lange Zeit andauern - wie lange, kann nicht vorhergesagt werden. Aber letztendlich lässt die Geldnachfrage nach und die Preise beginnen zu steigen, im Zeitablauf mit zunehmender Rate. Die Erwartung von Preissteigerungen wird zur selbsterfüllenden Prophezeiung. Zu Preiserhöhungen in Phase Zwei kommt es immer schneller, sobald mehr und mehr Bürger ihre Ausgaben beschleunigen, um der sinkenden Kaufkraft ihres Geldes entgegenzuwirken.

Phase Zwei geht dann in Phase Drei über, sobald die Wirtschaftssubjekte ihr Vertrauen in die zukünftige Kaufkraft ihres Geldes vollständig verlieren und die Geldnachfrage gegen Null geht. Keiner könne jedoch vorhersagen, wann genau die Phase Drei beginnt. In dieser finalen Phase versuchen die geldpolitischen Instanzen, den Vertrauensverlust in die Währung zu beenden, Selbst wenn sie drastische Maßnahmen einführen, um die Inflation zu beenden, bleiben sie erfolglos. Diese Phase wurde von Mises als ein sich selbst verstärkender „Crackup boom“ bezeichnet, da es große Kaufwellen gibt. Denn jeder möchte sein Geld in jegliche verfügbare Güter eintauschen (https://www.mises.ca/why-isnt-qe-causing-inflation/). 
Auf diese Argumentation setzen Vertreter und/oder Anhänger der ÖS traditionell die Vorhersage nahezu „sicher" aufkommender, stark anziehender Inflation durch die unkonventionelle Geldpolitik auf, ohne dies jedoch zeitlich zu konkretisieren. Dies bringt ihnen zu Recht regelmäßig den Vorwurf einer Selbst-Immunisierung ein. Denn Empirie wird so zur Spekulation. Ihr Rat ist für praktische Investitionszeiträume nicht zu gebrauchen, wenn ihre Prognosen nur über eine unbestimmt lange Zeitspanne betrachtet korrekt sind.

Bleibt die stark anziehende Inflation jedoch während praktischer Investitionszeiträume über 10 bis 15 Jahre aus, so wird von Vertretern der ÖS häufig argumentiert, man habe nicht mit $100 \%$ Sicherheit stark anziehende Inflation vorhergesagt, sondern nur gesagt, dies könne passieren. Dieses strategische Aussagemuster wird auch als ,fallacy of equivocation" bezeichnet, da man den Bedeutungsgehalt des Verbs „vorhersagen“ im Laufe der Debatte ändert. Eine weitere Verteidigungslinie lautet, die Autoren, die sogar eine Hyperinflation infolge unkonventioneller Geldpolitik vorhergesagt haben (French 2009; North 2012) ${ }^{1}$ seien gar keine genuinen „Austrians“. Üblicherweise wird diese Strategie als „no Scotsman fallacy“ bezeichnet, also als eine ,informal fallacy in which one attempts to protect a universal generalization from counterexamples by changing the definition in an ad hoc fashion to exclude the counterexample“ (https://en.wikipedia.org/wiki/No_true_Scotsman). Denn, betrachtet man ihre Homepages und Selbstauskünfte, so wird schnell deutlich, dass diese Autoren sehr wohl nach Prinzipien der ÖS argumentieren und handeln und dies auch explizit machen (Connell 2012).

Es wäre interessant zu erfahren, wie Israel und Hülsmann sich zu diesem augenfälligen und öffentlichkeitswirksamen Problem positionieren. Denn dies ist ein zentraler wunder Punkt in der Performance der ÖS seit dem Platzen der DotcomBlase. (Warum) Lagen Ron Paul, Peter Schiff, und Dutzende weiterer ,high-profile Austrian economists and investors" mit ihren auf der Theorie der ÖS basierenden Inflationsvorhersagen so unangenehm falsch (Connell 2012)? Ist dies lediglich ein Problem der Anwender der ÖS? Wie stehen Theoretiker der ÖS zur Vorhersage der Folgen der unkonventionellen Geldpolitik? (Warum) Enthalten sie sich einer Aussage? Oder argumentieren Theoretiker wie Bagus (2013) ähnlich wie die zuvor genannten Praktiker der ÖS: „Die Dynamik dieses Systems ist explosiv und führt direkt in die Hyperinflation“ und „Eine Lösung des Konstruktionsfehlers des Euro muss bald kommen, will man eine Hyperinflation noch vermeiden“"?

Im Falle der ,österreichischen“ Kritik an der unkonventionellen Geldpolitik setzt die Vorhersage höherer Inflation voraus, dass es den Notenbanken gelingt, auch die Zinsen am langen Ende zu drücken. Genau dies scheint aber weder für die Fed noch für die EZB nachhaltig der Fall zu sein (Belke et al. 2017; sowie die ECB and Its Watchers Conference 2018 in Frankfurt). Vielmehr zeigt sich, dass die Geldpolitik der Quantitativen Lockerung (QE) mittel- bis langfristig ineffektiv ist und somit die Inflationserwartungen auch nicht dauerhaft heben kann. Immerhin hatte die Fed doch schon 2014 ihr QE-Programm eingestellt, weil eine Bilanzverlängerung, also

\footnotetext{
1 Vgl. auch Ron Paul, 2011, in: https://www.thestreet.com/story/11041675/1/ron-paul-predicts-hyperinflation-says-dollar-will-be-rejected-as-reserve-currency-of-the-world.html und Peter Schiff in: http:// globaleconomicanalysis.blogspot.com/2009/01/peter-schiff-was-wrong.html.
} 
der Austausch von Nullzins-Assets gegen Nullzins-Assets, keine ersichtliche Wirkung hat. Allgemeiner ausgedrückt: trotz aller seit zehn Jahren anhaltender Notenbankakrobatik scheint die Inflationsrate einem White Noise-Prozess um eine leicht positive Konstante zu folgen (Miles et al. 2017).

Nun ändern Vertreter der ÖS systematisch leicht ihren Fokus und folgen so einer ,Moving the goal posts“-Strategie. Es handelt sich hierbei um eine Metapher „derived from goal-based sports that means to change the criterion (goal) of a process or competition while it is still in progress, in such a way that the new goal offers one side an intentional advantage“ ${ }^{\prime 2}$. Sie stellen sich auf den Standpunkt, die Inflation trete statt auf den Gütermärkten bereits auf den Vermögensmärkten auf, werde falsch gemessen, und deshalb unterschätzt. Sie liege in der Eurozone ,in Wahrheit" jetzt schon über der Zielrate. Unabhängig davon, ob das Argument der Falschmessung an sich stichhaltig ist ${ }^{3}$, legt man sich explizit darauf fest, dass man sich bereits in Phase Zwei der Mises'schen „monetary destruction“ befindet.

Mit anderen Worten: man argumentiert empirisch, um Positionen der ÖS zu fundieren. Dynamische Implikationen der dreistufigen Mises'schen ,,monetary destruction“" werden durch Inspektion der Datenlage fundiert. Bereits dies könnte deutlich im Widerspruch zu den weiter oben angeführten Grundprinzipien der ÖS stehen. Noch erstaunlicher ist, dass aus den empirischen Ergebnissen häufig auch konkrete wirtschaftspolitische Schlussfolgerungen gezogen werden. Beispielsweise wird ein sofortiger Ausstieg aus der unkonventionellen Geldpolitik gefordert. Dies insinuiert aber, dass der Übertritt des Systems in Stufe Drei nicht mehr weit entfernt ist.

Politisch verwundert, dass gerade die Vertreter der ÖS der EZB ,abkaufen“, sie wolle und könne mit unkonventioneller Geldpolitik (hier besonders QE) systematisch geldpolitische Wirkungen erzielen. Noch verwunderlicher ist, das man sich trotz starker Evidenz gegen die Effektivität dieser Maßnahmen empirisch konkret darauffestlegt, dass man bereits die Phase Zwei im Mises-Zyklus erreicht habe. In Bezug auf die US-amerikanischen Programme der Quantitativen Lockerung (QE) argumentierten Vertreter der ÖS teilweise sogar, diese könnten in Hyperinflation münden (siehe beispielsweise French 2009; North 2012; und Polleit 2014). ${ }^{4}$

Roubini (2015) bemerkt hierzu abwertend: „One result of this global monetarypolicy activism has been a rebellion among pseudo-economists and market hacks in recent years. This assortment of ,Austrian " economists, radical monetarists, gold bugs, and Bitcoin fanatics has repeatedly warned that such a massive increase in global liquidity would lead to hyperinflation, the US dollar's collapse, sky-high gold prices, and the eventual demise of fiat currencies at the hands of digital kryptocurrency counterparts. ... None of these dire predictions has been borne out by events“.

\footnotetext{
2 Vgl. https://en.wikipedia.org/wiki/Moving_the_goalposts.

3 Goolsbee und Klenow (2018) beispielsweise kommen zu dem Ergebnis, dass Inflation eher überschaetzt wird.

4 Siehe auch Ron Paul, 2011, in: https://www.thestreet.com/story/11041675/1/ron-paul-predicts-hyperinflation-says-dollar-will-be-rejected-as-reserve-currency-of-the-world.html. Vgl. darüber hinaus aus ÖS-Perspektive https://www.hayek-institut.at/der-billionenpoker/ oder https://mises.org/wire/falsepromises-behind-quantitative-easing und aus sozialisitischer Perspektive: http://socialdemocracy 21stcentury.blogspot.com/2014/03/did-austrians-never-predict.html.
} 
Ein Beharren der Vertreter der ÖS auf den von ihnen vertretenen Verlaufsmustern der Mises'schen „monetary destruction“ - trotz substanziell gegenläufiger empirischer Evidenz sowie der Bruch mit ihren eigenen Prinzipien durch den Versuch der Verifikation der eigenen Position durch die Empirie und die Ableitung konkreter wirtschaftspolitischer Implikationen - riskiert, dass die wirtschaftspolitischen Einlassungen der ÖS unglaubwürdig wirken. Beides trägt im Extremfall zu Zweifeln der Öffentlichkeit an der ökonomischen Disziplin als Ganzer bei. Deshalb sollten Vertreter der ÖS weniger apodiktisch argumentieren und ruhig mehr Mut bei der zielführenden Anwendung ökonometrischer Methoden und der Heranziehung von Ergebnissen stringenter ökonometrischer Studien offenbaren. Wie gerade gezeigt wurde, bedeutet dies keinesfalls, wie von Mises indirekt postuliert, den (endgültigen) Einstieg in die Planwirtschaft.

Eine ähnliche Argumentation ließe sich hier unter Berücksichtigung der NichtNeutralität des Geldes (Verteilungseffekte der Geldpolitik, Cantillon-Effekt nach Cantillon 2010) und der Erkenntnis, dass die Ausweitung von Bankkredit der wichtigste Treiber von von „Boom“ und „Bust“-Konjunkturzyklen ist, anführen (Mises 1912). Dies geschieht im folgenden Abschnitt in leicht verändertem Kontext an einem weiteren Fallbeispiel.

\subsection{Ultra-lockere Geldpolitik und gemeinschaftliche Haftung als Treiber politischer Instabilität}

Hayeks (1976a) „Geldtheorie und Konjunkturtheorie“ erklärt Konjunkturzyklen, also Aufschwung und Krise, mit Zentralbankfehlern. Vertreter der ÖS modifizieren diese Erklärung in der Regel um zwei Aspekte - die asymmetrische Zinssetzung (schnellere Zinssenkung in der Rezession als -erhöhung im Boom) und eine Blasenbildung auf Assetmärkten (siehe zum Beispiel Schnabl 2018). Anschließend werden diese Argumente für eine Warnung vor den zukünftigen Folgen unkonventioneller Geldpolitik genutzt und hieraus normative Schlussfolgerungen für die aktuelle und zukünftige Ausrichtung der Wirtschaftspolitik (hier der Geldpolitik) gezogen. Beides steht aber eigentlich in klarem Widerspruch zu den von Israel und Hülsmann klar herausgearbeiteten Grundsätzen der ÖS. Zu argumentieren, der Verweis auf Gefahren sei etwas anderes, weniger weitreichendes als die Prognose konkreter Folgen für die EWWU, reicht wohl nicht als Rechtfertigung.

Ein Anwendungsbeispiel ist der Euro-Aufruf vom 21. Mai 2018, in dem 154 Wirtschaftsprofessoren davor warnen, die europäische Währungs- und Bankenunion noch weiter zu einer Haftungsunion auszubauen (FAZ 2018). Auch Hülsmann als einer der beiden Autoren des hier zu diskutierenden Beitrags ist neben Bagus und vielen anderen der ÖS nahe stehenden Ökonomen dabei, sodass die Wahl des EuroAufrufs als Anwendungsbeispiel für die ÖS in der wirtschaftspolitischen Praxis voll gerechtfertigt erscheint.

Nicht nur die Passage „Die Haftungsunion unterminiert das Wachstum und gefährdet den Wohlstand in ganz Europa. Dies zeigt sich bereits jetzt in einem sinkenden Lohnniveau für immer mehr, meist junge Menschen ..." atmet geradezu den Geist der ÖS, indem sie auf dem Cantillon-Effekt und der dreistufigen Mises'schen „monetary destruction“ beruht. Zudem lässt sich an diesem Abschnitt des Aufrufs 
gut darlegen, dass Vertreter der ÖS viel stärker als bisher empirisch Farbe bekennen müssen, wenn Sie ihre Anliegen in konkrete Politik umsetzen wollen.

Starke Aussagen wie im Aufruf beinhalten zwar viel Theorie, provozieren aber die Infragestellung gleich mehrerer Teilargumentationsketten bzw. deren Elemente. Ein Beispiel hierfür ist die Teilpassage: „Dies zeigt sich bereits jetzt in einem sinkenden Lohnniveau für immer mehr, meist junge Menschen“. Gemeint ist hier wohl „,beginnend in Südeuropa und im Vergleich zu vorangegangenen Generationen“. Zu dem ,zeigt sich bereits jetzt“ erläutern einige Vertreter der ÖS nur, die derzeitigen realen Lohnerhöhungen seien nicht ernstzunehmen, da sie das Ergebnis einer Blase (unter Verweis auf Mises 1912) und nicht durch Produktivitätserhöhungen gedeckt seien (Schnabl 2018; Polleit 2014). Dies ist aber zunächst nicht mehr als eine Tatsachenbehauptung, ganz einfach konditioniert auf die Theorie nach Mises (1912).

Zudem erhebt sich natürlich seitens der interessierten Öffentlichkeit sofort die Frage, ob diese Interpretation zutrifft und wie man sie anhand der Daten erhärten würde. Will die ÖS in der öffentlichen Debatte bestehen, wird spätestens hier deutlich, dass sie in die „Untiefen“ der Empirie „hinabsteigen“ muss, um dem Vorwurf einer Immunisierung gegen Kritik und Falsifizierungsversuche entgegenzutreten und glaubwürdig zu bleiben.

Zugegebenermaßen handelt es sich hierbei allesamt um interessante Hypothesen, die den Nerv eines signifikanten Teils der deutschen Bevölkerung treffen dürften. Angesichts der Öffentlichkeit des Aufrufs ist davon auszugehen, dass sich die auf der Unterzeichnerliste vertretenden Anhänger der ÖS gut überlegt haben, wie man in empirischen Studien die im Aufruf klar enthaltenen Kausalitäten (wie z. B. „Blasen sind dominierende Treiber der Reallöhne“ oder ,die Haftungsunion ist Verursacher steigender Arbeitslosigkeit und fallender Löhne für jüngere Arbeitnehmer") identifizieren würde.

Eigentlich sollten derartige Verursachungsbehauptungen der reinen ÖS ja ohnehin schon entschieden zu weit gehen. Denn der positivistische Ansatz von Hypothesenbildung und -verwerfung im Lichte empirischen Datenmaterials in der Tradition von Mises wird - wie der Beitrag von Israel und Hülsmann klar herausarbeitet - für die Erklärung des menschlichen Handelns von den „Austrians“ eigentlich als unbrauchbar angesehen. Man könnte dies aber auch als Tendenz zur Selbst-Immunisierung gegen empirisch fundierte Kritik interpretieren.

Im Folgenden werden exemplarisch einige Bereiche genannt, wo es beim Versuch einer empirischen Identifikation der im Aufruf enthaltenen Kausalitäten durchaus Probleme geben könnte.

In Bezug auf Südeuropa lässt sich erstens festhalten, dass Spanien wegen des Strukturwandels in der Landwirtschaft und seines verkrusteten dualen Arbeitsmarktes schon in der 1980er und 1990er Jahren eine enorme Jugendarbeitslosigkeit aufwies, also lange bevor eine „Haftungsunion“ thematisiert wurde. Die gegenwärtige Periode im Gefolge der Eurokrise ist bereits das dritte Mal innerhalb von drei Jahrzehnten, dass Spanien unter einer Jugendarbeitslosigkeitsrate von über $40 \%$ leidet. Dolado et al. (2013) thematisieren folglich ein ,,dejà vu“ bei der spanischen Jugendarbeitslosigkeit. Ähnliches gilt als stilisiertes Faktum auch für andere südeuropäische Regionen. Gros (2013) spricht in diesem Zusammenhang aus Gründen verzerrter 
empirischer Erfassung sogar von Jugendarbeitslosigkeit als „Europe's Youth Unemployment Non-Problem“.

Zweitens hat die Verschlechterung der Lage am Arbeitsmarkt der vergangenen Jahre zumindest kurzfristig auch mit der von der Troika durchgesetzten Politik der Schuldenreduzierung und internen Abwertung zu tun. Fallende Löhne sind also zentraler Bestandteil der Lösung und eben nicht das Problem; ein mehrere Jahre andauernder U-Turn der Löhne ist ein stilisiertes Faktum fast aller Strukturreformen (neben vielen anderen siehe Alcidi et al. 2016). Wenn die Vertreter des Aufrufs wirklich über die dort fallenden Löhne besorgt wären, müssten die Unterzeichner eigentlich die Pläne der Kommission begrüßen - also das Gegenteil dessen, was sie fordern. Diese strebt ja die Glättung asymmetrischer Schocks an, ohne dass es dabei zu kurzfristigen Beschäftigungseinbrüchen, die sich bei Hysterese sogar verstetigen können, kommt (Belke 2013).

In Bezug auf Deutschland kommt die Arbeitsmarktforschung zu dem Ergebnis, dass die Reallohnentwicklung der letzten anderthalb Jahrzehnte vor allem auf die Auswirkungen von Hartz IV auf das Verhalten der Gewerkschaften in Tarifverhandlungen zurückzuführen ist. Die Jahre von 2005 bis 2008 waren genau die Jahre, in denen die Nominallöhne praktisch stagnierten, so dass sich bei wachsender Produktivität die Wettbewerbsfähigkeit deutscher Unternehmen verbesserte und die Arbeitslosigkeit reduziert wurde (IMF, 2017, S. 77; Burda und Seele 2016). In diesen Jahren war jedoch eine Haftungsunion überhaupt noch nicht absehbar. Auch hier hat der Aufruf ein gravierendes empirisches Identifikationsproblem, denn das „Counterfactual“ (siehe Abschn. 3.2) zeichnet sich durch eine ähnlich verhaltene Lohnentwicklung aus wie die Periode des „Treatment“" mit deiner Haftungsunion.

Schließlich verweisen „Austrians“ tendenziell darauf, dass die Immobilienpreise so hoch sind, dass sie eine fallende Kaufkraft der Jüngeren begründen können. Dabei wäre allerdings zu berücksichtigen, dass der Anteil der Bevölkerung, die in eigenen Immobilien wohnt, in Deutschland schon immer vergleichsweise niedrig war. Es wäre auch mit einzubeziehen, dass bei den heutigen Zinssätzen die mit der Aufnahme einer Hypothek verbundenen Belastungen deutlich niedriger sind, als sie es etwa in den 1980er oder 1990er Jahren waren (Belke und Gros 2014). Schließlich müsste man in der Lage sein, Blasen exakt zu identifizieren etc.

Auch kann der Rückgang der Produktivitätszuwächse durch Treiber einer säkularen Stagnation oder durch die Geldpolitiken bedingt sein. Beide Seiten haben ihre Argumente, die ausgiebig und wiederholt ausgetauscht wurden. Es kann dann aber nur der Empirie obliegen, den dominanten Treiber (nach Vorstellung der Vertreter der ÖS ist dies die Geldpolitik) zu identifizieren.

Schließlich müsste Auskunft darüber gegeben werden, wie man sich die konkrete zeitliche Zuordnung der verschiedenen im Aufruf genannten kausalen Effekte genau vorstellt.

Israel und Hülsmann fassen in diesem Zusammenhang eine Reihe von Konsequenzen eienr Geldmengenausweitung und Zinssenkung vom analytischen Standpunkt der ÖS zusammen und schlussfolgern: „Diese entsprechen ganz offensichtlich nicht den erklärten Zielen der Geldpolitik. Sowohl die dargelegten Umverteilungseffekte, erhöhte Verschuldung, systemisches Risiko, als auch Wirtschaftskrisen, stellen vielmehr Probleme dar, die es aus wirtschaftspolitischer Sicht zu lösen gilt“. Dies 
ist ebenso vollkommen richtig. Nur: es handelt sich dabei deutlich nicht um ein Alleinstellungsmerkmal der ÖS. Das Argumentationsmuster gehört samt der Vorhersage geldpolitisch ausgelöster Interventionsspiralen (Finanzmarktregulierung etc.) vielmehr bereits unabhängig von der Mises-Lektüre zum Standard-Werkzeugkasten eines „Mainstream“-Makroökonomen (für eine Übersicht hierzu vgl. Belke und Verheyen 2014).

Interessanter wäre es gewesen, wenn die Verfasser den expliziten Einfluss von Mises und anderen Vertretern der ÖS auf die theoretische Literatur nachgewiesen hätten, auf die sich die „Mainstreamer“, die die (nicht) intendierten Nebeneffekte einer ultra-expansiven Geldpolitik thematisieren, beziehen. Analoges gilt auch für andere Ausführungen der Verfasser wie zum Beispiel zur Phillipskurvendebatte.

Um die im Euro-Aufruf getroffenen Tatsachenbehauptungen zu belegen, dürften die Unterzeichner des Aufrufs, darunter auch Vertreter der ÖS („New Austrians“) also noch einiges nachzuholen haben. Natürlich könnten sie sich theoretisch auf den Standpunkt zurückzuziehen, die im Aufruf angeführten kausalen Aussagen gälten nur ceteris paribus; es gebe auch andere Ursachen für steigende Arbeitslosigkeit und fallende Löhne. Dies bedeute auch keinesfalls, dass die dem Aufruf zugrunde liegende Argumentation falsch sei: eine Falsifizierung der Hypothesen der ÖS sei eben nicht möglich (Polleit 2017).

Eine derartige Haltung könnte aber genau die oben angesprochene, für die Wirkung der ÖS in der Öffentlichkeit fatale Selbst-Immunisierung gegen Kritik anderer Denkschulen ausdrücken. Zwar nimmt die ÖS, wie es Israel und Hülsmann ja auch richtig zum Ausdruck bringen, beim verbal-logischen Vorgehen der ÖS grundsätzlich in Kauf, dass es mehr Raum für Ungenauigkeiten und Missverständnisse gibt. Begibt man sich aber selber in die Offensive, diagnostiziert öffentlichkeitswirksam, dass sich eine Volkswirtschaft bereits in einer konkreten Stufe des Mises'schen „destruction“ befinde, und gibt dann noch auf der Grundlage der ÖS konkrete Politikempfehlungen, ergibt sich die logische und strategische Pflicht, empirisch korrekt und konkret datenorientiert zu arbeiten.

Bei empirischen Behauptungen sollte man sich auch als „Österreicher“ grundsätzlich auf die Daten beziehen, über die man eine Aussage macht, wie in unserem Beispiel über die derzeitigen Lohnsenkungen. Und wenn man argumentiert, dass diese Daten nicht aussagekräftig sind, weil die Löhne derzeit durch eine nicht nachhaltige Blase getrieben werden, dann sollte die Aussage entweder ganz unterlassen oder aber die Konditionierung auf die zugrunde gelegte Theorie (Hellwig 2007, S. 12) deutlich gemacht werden. Wenn man aber nicht weiss, wissen kann oder wissen will (wie Vertreter der ÖS ja vorgeben), ob und mit welcher Wahrscheinlichkeit diese Theorie mit dem datengenerierenden Prozess übereinstimmt, suggeriert ein derartiger Aufruf eine nicht vorhandene Eindeutigkeit im Hinblick auf zentrale Kausalbeziehungen.

Eine ähnliche Argumentation ließe sich für eine zweite kritische Aussage des Aufrufs anführen: „Wenn der Europäische Stabilitätsmechanismus (ESM) wie geplant als Rückversicherung für die Sanierung von Banken (Backstop) eingesetzt wird, sinkt für Banken und Aufsichtsbehörden der Anreiz, faule Kredite zu bereinigen“. In Bezug auf die behauptete Verschleppung der Bereinigung von Krediten wäre wie im ersten Fallbeispiel ein gründlicher Blick in die konkreten Daten erforderlich. 
Eine derartige Inspektion der Vorgeschichte würde rasch zeigen, dass die Anreize zur Bereinigung schon auf nationaler Ebene bisher nicht vorhanden waren und die Bereinigung, soweit sie überhaupt erfolgt ist, von der europäischen Aufsicht erzwungen wurde. Darüber hinaus ist in die Argumentation einzubauen, dass die nationalen Instanzen die europäische Aufsicht daran gehindert haben, bei der Bereinigung der faulen Kredite schneller vorzugehen (Hellwig 2017a, 2017b). Beides kam nicht überraschend und lässt sich aus polit-ökonomischen Analysen als anreizkonform ableiten (Belke et al. 2016).

Auch wäre in der Argumentation zu berücksichtigen, dass die Beihilfekontrolle der Kommission dafür gesorgt hat, dass es anders als bei den deutschen Bankensanierungen (HSH Nordbank) bei Monte dei Paschi, der Banca Popolare di Vicenza und der Veneto Banca sowie auch bei der Banco Popular Español jeweils ein Bailin der nachrangigen Gläubiger gab. ${ }^{5}$

Es sei denn, die oben genannte kritische Aussage des Aufrufs wäre konditioniert auf eine/die Theorie der ÖS - zum Beispiel auf die Theorie, dass eine Zentralisierung auf europäischer Ebene hinsichtlich der Wohlfahrtswirkungen immer (auch nicht bei externen Effekten und öffentlichen Gütern) dem Subsidiaritätsprinzip und der nationalen Souveränität unterlegen ist.

\subsection{Zur Reichweite des ,österreichischen“"Ansatzes}

„New Austrians“ argumentieren, mit etwas mehr Worten und Zeit ließen sich auch aufwendigere ökonomische Aussagen logisch-deduktiv aus der Logik des menschlichen Handelns gewinnen (für einen Überblick vgl. Polleit 2017): dass zum Beispiel der Sozialismus als Wirtschaftssystem nicht durchführbar ist; oder dass die Vermehrung der Geldmenge durch Kreditvergabe, die nicht nur durch echte Ersparnis gedeckt ist, notwendigerweise zu wirtschaftlichen Störungen führt; oder dass ein Vermehren der Geldmenge die Volkswirtschaft nicht reicher macht; oder dass der Urzins aus handlungslogischen Gründen nicht Null, geschweige denn negativ sein kann (Böhm-Bawerk). All diese Aussagen könnten wir mit Gewissheit machen. Denn die gerade angeführten Aussagen ließen sich nicht widerspruchsfrei verneinen (Polleit 2017).

Der Beweis aber, dass sie sich als unwiderlegbare Wahrheiten (,irrefutable truths") im Sinne Immanuel Kants verkaufen lassen (Ökonomik als Praxeologie, Mises 1940), muss gerade für den Bereich der Geldtheorie und -politik aber erst noch im Verlauf eines voraussichtlich sehr langen Wegs geführt werden. Wissenschaftlich anerkannte erfolgreiche Belege in dieser Hinsicht sind bisher nicht bekannt. Es wurde lediglich behauptet, dass dies möglich sei. Bis dahin erweisen die Ökonometrie, die Statistik und eine vorsichtige und umsichtige Auswertung der Datenlage unverzichtbare Dienste, die oben genannten Hypothesen im Sinne Poppers vorläufig nicht abzulehnen (Popper 1935).

\footnotetext{
5 Vgl. Hellwig (2017a) und http://www.handelsblatt.com/finanzen/banken-versicherungen/veneto-bancaund-banca-popolare-di-vicenza-rettungsversuch-mit-zaehneknirschen/.
} 
Der Titel des hier diskutierten Beitrags „Die Österreichische Schule als Gegenprogramm zur Standardökonomik" sollte also insgesamt gesehen mit einem Fragezeichen versehen werden. Das Korreferat hat versucht aufzuzeigen, dass der Ansatz der ÖS in vielerlei Hinsicht kaum von dem Teil des „Mainstreams“ abweicht, der systematischen Interventionen von Staat und Zentralbank in den Wirtschaftskreislauf skeptisch gegenüber steht, und wegen interner logischer Widersprüche sogar hinter diesen zurückzufallen droht.

Die Autoren schreiben: „Die ökonomische Theorie im Sinne der ÖS stellt aber keine Sätze dieser Art auf. Sie stellt generell keine Hypothesen über die zukünftige Entwicklung empirischer Variablen auf.“ Wenn man ,nichts wissen kann“, aus der Historie nichts für die Zukunft extrapolieren kann und keine Folgewirkungen verschiedener Politikalternativen ableiten kann, warum und auf welcher methodischen Grundlage mischen sich Vertreter der ÖS dann doch immer wieder in die Politikberatung ein? Es wurden zudem wichtige „,Marketing “-Probleme der ÖS gerade in den Bereichen identifiziert, in denen die ÖS ein Alleinstellungsmerkmal nutzen könnte, und im Bereich der empirischen Unterfütterung Vorschläge für ein geeignetes zukünftiges Forschungsprogramm der ÖS gemacht.

Es wäre sehr nützlich, wenn sich die regelmäßigen Mahner und lautstarken Warner aus der Gruppe der ÖS einmal systematisch mit der Treffsicherheit Ihrer eigenen „Vorhersagen des Untergangs“ in der Vergangenheit beschäftigen würden - in den letzten zehn Jahren seit der jüngsten Weltfinanzkrise, aber auch schon in der Phase nach dem Platzen der Dotcom-,,Blase“. Dies gilt vor allem in Bezug auf die Auswirkungen der unkonventionellen Geldpolitik auf die Inflation und die dynamische Ausprägung einzelner Makrovariablen wie der Reallöhne und Investitionen im Rahmen der Erklärung von Booms und Pleiten im Rahmen der „Österreichischen“ Konjunkturtheorie. Es würde vielleicht helfen, zu jener Bescheidenheit zurückzufinden, die der von der ÖS zurecht immer wieder angemahnten Begrenztheit unseres Wissens angemessen ist.

\section{Fazit}

Vom Selbstverständnis her sehen sich viele Vertreter der ÖS heute der Heterodoxie zugehörig, die sich aber als Teil eines neu entstehenden heterodoxen, d.h. nicht neoklassischen Mainstreams versteht (Koppl 2006). Man sollte jedoch bei der inhaltlichen Einordnung der teilweise zu stark formulierten Abgrenzungen der „New Austrians“" von der Neoklassik und vom „Mainstream“ bedenken, dass die Übertreibung der Differenzen auch als bewusste Strategie gesehen werden kann, die lediglich die Kohärenz einer marginalisierten Perspektive als alternatives Forschungsparadigma stärken soll (Backhouse 2004).

Denn wie mein Korreferat zeigt, sind die inhaltlichen Unterschiede zwischen den „Austrians“ und denjenigen Vertretern des „Mainstreams“, die interventionsskeptisch sind, in relevanten Themenbereichen wie der monetären Ökonomik und der Konjunkturtheorie kaum identifizierbar. Dies gilt zumindest dann, wenn man ehrlichkeitshalber von der Strohpuppe „Mainstream“ absieht oder diesen in die beiden zahlenmäßig signifikant besetzten Lager unterteilt: den grundsätzlich interventions- 
freundlichen (Neukeynesianischen) und den eher interventionsskeptischen „Mainstream“. Im Korreferat wurde eine große inhaltliche Schnittmenge der ÖS mit dem zweiten Lager identifiziert.

Es werden zudem logische Inkonsistenzen und ein Marketing-Problem der „New Austrians“ diagnostiziert. Denn sie fordern auf der Grundlage recht allgemein gehaltener historischer Daten und Zusammenhänge Politikänderungen, identifizieren teilweise sogar die genaue Phasen-Position Europas im Rahmen der dreistufigen Mises'schen ,monetary destruction“, immunisieren sich aber gleichzeitig oft gegen die Bringschuld exakter empirischer Belege hierfür. Dies schafft ein nicht zu vernachlässigendes Glaubwürdigkeitsproblem.

Das Aufkommen des Internets spielt den „New Austrians“ allerdings in die Karten. Dass sie die Finanzkrise grundsätzlich, wenn auch nicht zeitscharf, vorhergesagt haben und auch die ultra-lockere Geldpolitik der Zentralbanken scharf kritisieren, hat ihnen mehr Aufmerksamkeit gebracht, ,als jemals seit den Lebzeiten von Menger und Mises“. Dazu kommt, dass die auf den Prinzipien der österreichischen Geldtheorie basierende Internetwährung Bitcoin die Ideen der alten Österreicher heute einer ganz neuen Generation näher bringt (Jilch 2016).

$\mathrm{Zu}$ Beginn dieses Korreferats wurde argumentiert, dass die „Österreicher“ klassisch liberal, aber nicht „,neoliberal“ seien. Zudem hatten sie mit vielen ihrer Warnungen Recht und wurden dennoch zumeist ignoriert (Jilch 2016). Das hatte lange einen entscheidenden Vorteil: sie wurden auch nie von einer politischen Ideologie in Geiselhaft genommen. Mittlerweise aber werden Ökonomen der ÖS gerade wegen ihrer ,starken“, aber nicht oder widersprüchlich empirisch detailliert belegten Aussagen über die zukünftigen Folgen wirtschaftspolitischer Maßnahmen immer häufiger politisch vereinnahmt. Eingeschränkte Unabhängigkeit aber würde den Fortbestand der ÖS als wissenschaftlich seriöse Denkschule gefährden. Ein wichtiger Pfeiler in der Koalition der Denkschulen gegen eine zu starke staatliche Lenkung im Wirtschaftsgeschehen ginge verloren.

\section{Literatur}

Abadie, A., Diamond, A., \& Hainmueller, J. (2014). Comparative politics and the synthetic control method. American Journal of Political Science, 59(2), 495-510.

Akerlof, G. A., Dickens, W.T., \& Perry, G.L. (1996). The macroeconomics of low inflation. Brookings Papers on Economic Activity. https://doi.org/10.2307/2534646.

Alcidi, C., Belke, A., Giovannini, A., \& Gros, D. (2016). Macroeconomic adjustment programmes in the Euro area: an overall assessment. International Economics and Economic Policy, 13(3), 345-358.

Backhouse, R.E. (2004). A suggestion for clarifying the study of dissent in economics. Journal of the History of Economic Thought, 26(2), 261-271.

Bagus, P. (2013). Kann der Euro überleben?, Ludwig von Mises Institut Deutschland. https://www. misesde.org/?p=4635 (erstellt am 25. März 2013, abgerufen am 15.Febr. 2019).

Bagus, P. (2016). Warum die „Austrians“ keine Neoliberalen sind, Ludwig von Mises Institut Deutschland. https://www.misesde.org/?p=11901 (erstellt am 1. Febr. 2016, abgerufen am 15. Febr. 2019).

Bailey, M. J. (1956). The welfare cost of inflationary finance. Journal of Political Economy, 64, 93-110.

Basse, T. (2006). An Austrian version of the Lucas critique. Quarterly Journal of Austrian Economics, 9(1), 15-26.

Belke, A. (2000). Political business cycles in the German labour market? Empirical tests in the light of the Lucas-critique. Public Choice, 104, 225-283.

Belke, A. (2013). Towards a genuine economic and monetary union-comments on a roadmap. Politics and Governance, 1(1), 48-65. 
Belke, A. (2014). „Troika Assessment“ - Zur künftigen Rolle des IWF in Europa, Ökonomenstimme. http://www.oekonomenstimme.org/artike1/2014/03/troika-assessment--zur-kuenftigen-rolle-desiwf-in-europa/ (erstellt am 12. März 2014, abgerufen am 15. Febr. 2019).

Belke, A. (2018). Helicopter money: should central banks rain money from the sky? Intereconomics - Review of International Trade and Development, 53(1), 34-40.

Belke, A., \& Gros, D. (2014). Kontraproduktive unkonventionelle Geldpolitik? oder: Wie das Gespenst der Deflation nicht zu vertreiben ist. Ökonomenstimme, 24. April.

Belke, A., \& Polleit, T. (2010). Monetary economics in globalised financial markets. Heidelberg, Berlin, New York: Springer.

Belke, A., \& Verheyen, F. (2014). The low interest rate environment, global liquidity spillovers and challenges for monetary policy ahead. Comparative Economic Studies, 56(2), 313-334.

Belke, A., Beckmann, J., \& Kühl, M. (2011). The stability of the dollar-euro exchange rate determination equation-a time-varying coefficient approach. Review of World Economics, 147(1), 11-40.

Belke, A., Dobrzanska, A., Gros, D., \& Smaga, P. (2016). (When) should a non-euro country join the banking union? Journal of Economic Asymmetries, 14, 4-19.

Belke, A., Göcke, M., \& Werner, L. (2014). Hysteresis effects in economics-different methods for describing economic path-dependence. In J.C. Dias (Hrsg.), Hysteresis-types, applications and behavior patterns in complex systems (S. 19-42). New York: Nova.

Belke, A., Gros, D., \& Osowski, T. (2017). The effectiveness of the Fed's quantitative easing policy: new evidence based on interest rate differentials. Journal of International Money and Finance, 73, 335-349.

Block, W. (2005). Rejoinder to Caplan on Bayesian economics. Journal of Libertarian Studies, 19(1), 79-95.

Burda, M., \& Seele, S. (2016). No role for the Hartz reforms? Demand and supply factors in the German labor market, 1993-2014. SFB 649 discussion paper 2016-010. Berlin: Humboldt University.

Campos, N. F., Coricelli, F., \& Moretti, L. (2014). Economic growth and political integration: estimating the benefits from membership in the European Union using the synthetic counterfactuals method. IZA discussion paper, Bd. 8162. Bonn: Institute for the Study of Labor.

Cantillon, R. (2010). An essay on economic theory, an English translation of the author's Essai sur la Nature du Commerce en Général. Auburn: Ludwig von Mises Instirute. Erstveröffentlichung 1755

Caplan, B. (2003). Probability and the synthetic a priori: a reply to Block. Quarterly Journal of Austrian Economics, 6(3), 77-83.

Charalambakis, J. (2016). Market hysteresis and capitulation: financial stress and secular trends, Austrian Economics Center, Vienna. https://www.austriancenter.com/market-hysteresis-and-capitulationfinancial-stress-and-secular-trends/ (erstellt am 3. Febr. 2016, abgerufen am 16.02.2019).

Christ, C.F. (1993). Assessing applied econometric results. Federal Reserve Bank of St. Louis Review, 75(2), 71-94.

Connell, S. (2012). Is the Austrian school wrong about inflation? https://seekingalpha.com/article/651641is-the-austrian-school-wrong-about-inflation (erstellt am 1. Juni 2012, abgerufen am 16. Febr. 2019).

Dolado, J. J., Felgueroso, F., \& Jansen, M. (2013). Intereconomics. Review of European Economic Policy, 48(4), 209-215.

Dowd, K. (1994). The costs of inflation and Disinflation. Cato Journal, 14(2), 305-331.

FAZ (2018). Aufruf - Der Euro darf nicht in die Haftungsunion führen!, Frankfurter Allgemeine Zeitung. http://www.faz.net/aktuell/wirtschaft/eurokrise/oekonomen-aufruf-euro-darf-nicht-inhaftungsunion-fuehren-15600325.html (erstellt am 21. Mai 2018, abgerufen am 16. Febr. 2019).

French, D. (2009). Store 'em if you got 'em, Mises daily articles. https://mises.org/library/store-em-ifyou-got-em (erstellt am 17. Aug. 2009, abgerufen am 16. Febr. 2019).

Friedman, M. (1960). A program for monetary stability. New York: Fordham University Press.

Galí, J. (2008). Monetary policy, inflation, and the business cycle: an introduction to the new Keynesian framework. Princeton, Oxford: Princeton University Press.

Galí, J., \& Gertler, M. (2007). Macroeconomic modeling for monetary policy evaluation. Journal of Economic Perspectives, 21(4), 25-45.

Garrison, R. W. (1986). From Lachmann to Lucas: on institutions, expectations, and equilibrating tendencies. In I. M. Kirzner (Hrsg.), Subjectivism, intelligibility and economic understanding (S. 87-101). Houndmills, Basingstoke and London: Macmillan.

Garrison, R. W. (2018). Mises and his methods, Mises Wire. https://mises.org/wire/mises-and-his-methods (erstellt am 20. Febr. 2018, abgerufen am 16. Febr. 2019).

Georgescu-Roegen, N. (1971). The entropy law and the economic process. Cambridge: Harvard University Press. 
Goolsbee, A. B., \& Klenow, P.J. (2018). Internet rising, prices falling: measuring inflation in a world of e-commerce. NBER working paper 24649. Cambridge: National Bureau of Economic Research.

Granger, C. W. J. (1991). Developments in the study of cointegrated variables. In R.F. Engle \& C.W.J. Granger (Hrsg.), Long-run economic relationships: readings in cointegration (S. 65-80). Oxford: Oxford University Press.

Granger, C.W.J. (1999). Empirical modeling in economics: specification and evaluation. Cambridge: Cambridge University Press.

Granger, C. W. J. (2003). Some aspects of causal relationships. Journal of Econometrics, 112, 69-71.

Granger, C. W. J., \& Newbold, P. (1977). Forecasting economic time series (2. Aufl.). Amsterdam: Elsevier. de Grauwe, P. (2010). The scientific foundation of Dynamic Stochastic General Equilibrium (DSGE) models. Public Choice, 144(3-4), 413-443.

Gros, D. (2013). Europe's youth unemployment non-problem, Project Syndicate. https://www.projectsyndicate.org/commentary/the-mirage-of-high-youth-unemployment-in-europe-by-daniel-gros (erstellt am 6. Juni 2013, abgerufen am 16. Febr. 2019).

Hagemann, H., Nishizawa, T., \& Ikeda, Y. (2010). Austrian economics in transition. Houndsmille: Palgrave Macmillan.

von Hayek, F. A. (1935). Prices and production. Auburn: Ludwig von Mises Institute.

von Hayek, F. A. (1976a). Geldtheorie und Konjunkturtheorie (2. Aufl.). Salzburg: Wolfgang Neugebauer Salzburg. Erstveröffentlichung 1929.

von Hayek, F. A. (1976b). Denationalisation of money: an analysis of the theory and practice of concurrent currencies. Hobart paper special 70. London: The Institute of Economic Affairs.

Hellwig, M. (2007). Wirtschaftspolitik als Rechtsanwendung: zum Verhältnis von Jurisprudenz und Ökonomie in der Wettbewerbspolitik. Max Planck Institute for Research on Collective Goods, working paper 19. Bonn: Max Planck Institute for Research on Collective Goods.

Hellwig, M. (2017a). Precautionary Recapitalisations-Time for a Review, In-Depth analysis. European Parliament, PE 602.089. July: Brussels.

Hellwig, M. (2017b). Competition Policy and Sector-Specific Regulation in the Financial Sector. OECD Workshop on Competition and Regulation Paris, 04.12.2017.

Holcombe, R. (2015). Public choice and Austrian economics. In C. J. Coyne \& P. J. Boettke (Hrsg.), The Oxford handbook of Austrian economics. https://doi.org/10.1093/oxfordhb/9780199811762.013.21.

Hoppe, H.-H. (1997). On certainty and uncertainty, or: How rational can our expectations be? Review of Austrian Economics, 10(1), 49-78.

Horwitz, S. (2003). The costs of inflation revisited. Review of Austrian Economics, 16(1), 77-95.

Israel, K.-F. (2016). The „Lucas critique“ is Misesian at its core, Mises wire. https://mises.org/wire/lucascritique-misesian-its-core (erstellt am 30. Aug. 2016, abgerufen am 18. Febr. 2019).

Jilch, N. (2016). Ökonomie: Die Außenseiter aus Österreich. Die Presse, 26. Oktober.

Keynes, J. M. (1936). The general theory of employment, interest and money. New York: Harcourt, Brace.

Knight, F. F. (1964). Risk, uncertainty and profit. New York: Century. Erstveröffentlichung 1921

Koop, G. (2017). Bayesian methods for empirical macroeconomics. Review of Economic Analysis, Rimini Centre for Economic Analysis, 9(1), 33-56.

Koppl, R. (2006). Austrian economics at the cutting edge. Review of Austrian Economics, 19(4), 231-241.

Leroy, S. F. (1992). On policy regimes. In K. D. Hoover (Hrsg.), Macroeconometrics: developments, tensions and prospects (S. 235-251). Boston: Kluwer.

Lucas, R.E. (1976). Econometric policy evaluation: a critique. Carnegie-Rochester conference series on public policy, Bd. 1 (S. 19-46).

McCallum, B.T. (1982). Macroeconomics after a decade of rational expectations: some critical issues. Federal Reserve Bank of Richmond Economic Review, 68(6), 3-12.

Miles, D., Panizza, U., Reis, R., \& Ubide, Á. (2017). And yet it moves-inflation and the great recession. Geneva reports on the world economy 19. Geneva: International Center for Monetary and Banking Studies.

von Mises, L. (1912). The theory of money and credit. Auburn: Ludwig von Mises Institute.

von Mises, L. (1940). Nationalökonomie: Theorie des Handelns und Wirtschaftens. Genf: Editions Union.

von Mises, L. (1962). The ultimate foundation of economic science-an essay on method. Princeton, New Jersey, Toronto, London, New York: D. Van Nostrand Company, Inc.

von Mises, L. (1977). Comments about the „mathematical treatment of economic problems“. Journal of Libertarian Studies, 1(2), 97-100.

von Mises, L. (1981). Preface to the English edition. In The theory of money and credit. Erstveröffentlichung 1934.

von Mises, L. (1996). Human action (4. Aufl.). San Francisco: Fox \& Wilkes. 
North, G. (2012). How to end the Fed, and how not to, Mises Daily Articles. https://mises.org/library/howend-fed-and-how-not (erstellt am 10. Sept. 2012, abgerufen am 17. Febr. 2019).

Olesen, F. (2010). Uncertainty, bounded rationality and post-Keynesian macroeconomics. European Journal of Economics and Economic Policies, Intervention, 7(1), 109-124.

Olesen, F. (2016). The Lucas critique-is it really relevant? Working paper no. 7, Department of Business \& Management, Macroeconomic Methodology, Theory and Economic Policy (MaMTEP), Aalborg University, Denmark.

Polleit, T. (2014). There will be (hyper)inflation, Mises Daily Articles, Mises Institute. https://mises.org/ library/there-will-be-hyperinflation (abgerufen am 16. Febr. 2019)

Polleit, T. (2017). Über die Logik des Handelns, Antrittsvorlesung, Bayreuth. https://www.misesde.org/? $\mathrm{p}=16485$ (erstellt am 22. Juni 2017, abgerufen am 19. Febr. 2019).

Popper, K. R. (1935). Logik der Forschung. Wien: Springer.

Romer, P. (2016). The trouble with macroeconomics, Stern School of Business, New York University, 14 September. forthcoming in: The American Economist

Rothbard, M. N. (2010). The mystery of banking (2. Aufl.). Auburn: Ludwig von Mises Institute.

Roubini, N. (2015). Are unconventional monetary policies here to stay? https://www.weforum.org/agenda/ 2015/02/are-unconventional-monetary-policies-here-to-stay/ (abgerufen am 19. Febr. 2019).

Sargent, T.J. (1979). Estimating vector autoregressions using methods not based on explicit economic theories. Federal Reserve Bank of Minneapolis Quarterly Review, 3, 8-15.

Schnabl, G. (2018). Wie man mit Hayek den Zusammenhang zwischen ultra-lockerer Geldpolitik und politischer Instabilität erklären kann, Wirtschaftliche Freiheit. http://wirtschaftlichefreiheit.de/ wordpress/?p=22022 (erstellt am 12. Jan. 2018, abgerufen am 19. Febr. 2019).

Selgin, G. A. (1990). Monetary equilibrium and the productivity norm of price-level policy. Cato Journal, $10(1), 265-287$.

Selgin, G. A. (1997). Less than zero: the case for a falling price level in a growing economy. Hobart paper 132. London: Institute for Economic Affairs.

Shmelev, S., \& Shmeleva, I. (2012). Sustainability analysis - an interdisciplinary approach. New York: Palgrave MacMillan.

Skott, P. (2014). Pluralism, the Lucas critique, and the integration of macroeconomics and microeconomics'. Review of Political Economy, 26(4), 503-515.

Smets, F., \& Wouters, R. A.F. (2003). An estimated dynamic stochastic general equilibrium model of the Euro area. Journal of the European Economic Association, 1(5), 1123-1175.

Woodford, M. (2003). Interest and prices: foundations of a theory of monetary policy. Princeton, Oxford: Princeton University Press. 